\title{
Out-of-Plane Deformation Analysis of the Thin-Walled Closed Curved Box Girder under the Temperature Gradient
}

\author{
Zeying Yang, ${ }^{1}$ Chenghe Wang, ${ }^{1}$ Yinglin Sun ${ }^{D},{ }^{1}$ Yangyudong Liu, ${ }^{1}$ Zhengquan Cheng, \\ and Weisong $\mathbf{Q} \mathbf{u}^{2}$ \\ ${ }^{1}$ School of Civil Engineering, Shandong University, Jinan 250061, Shandong, China \\ ${ }^{2}$ School of Mechanical and Electrical Engineering, Shandong Jianzhu University, Jinan 250101, Shandong, China \\ Correspondence should be addressed to Yinglin Sun; 1916878460@qq.com
}

Received 19 August 2021; Accepted 25 September 2021; Published 16 October 2021

Academic Editor: Luigi Fenu

Copyright (c) 2021 Zeying Yang et al. This is an open access article distributed under the Creative Commons Attribution License, which permits unrestricted use, distribution, and reproduction in any medium, provided the original work is properly cited.

\begin{abstract}
For calculating the thin-walled closed curved box girder caused by the temperature gradient of the internal force and displacement, based on the fundamental differential equation of the curve beam and the principle of minimum energy, set a reverse statically indeterminate simply supported curve beam as the basic structure, consider the warping effect of the closed curve box girder, and put forward a kind of plane curve beam temperature deformation simple analytical calculation method. Compared with the finite element calculation results, the relative error of the analytical calculation results is less than $5 \%$. It is concluded that the analytical method has sufficient accuracy in calculating the out-of-plane deformation of the thin-walled closed curved box girder under the temperature gradient.
\end{abstract}

\section{Introduction}

In recent years, with the construction of urban overpasses, curved beams are increasingly used in modern engineering structures. Scholars at home and abroad have studied the coupling characteristics of bending and torsion of curved beams [1-6] and the factors such as warping, distortion, and shear lag of thin-walled closed curved box girders [7-10]. Temperature effect is one of the main causes of bridge engineering problems. The research on the temperature effect of bridges mainly focuses on straight bridges [11-13], and the research on the temperature effect of curved beams is less. Literature studies [14-18] used the finite element method to analyze the stress and deformation of the curved beam under temperature load, and literature studies [19-22] used the analytical method to analyze the temperature effect of the curved beam but do not consider the warping effect of the curved beam under temperature in the out-of-plane direction.

Based on the basic differential equation of the curved beam and the principle of minimum potential energy, this paper presents a primary torsional statically indeterminate simply supported curved beam as the basic structure. A simple analytical method for calculating the vertical temperature gradient deformation of plane curved beams is presented.

\section{Deformation of the Simply Supported Statically Indeterminate Curved Beam under the Vertical Temperature Gradient}

2.1. Basic Assumptions. To analyze the temperature effect of the thin-walled closed curved box girder, the following basic assumptions are adopted:

(1) Warping deformation is only considered comprehensively with vertical bending deformation

(2) The strain and strain curvature of the curved box girder are considered as curved beams with small curvature, and the deformation is in a small deformation range 
(3) Distortion of the cross section is not considered

(4) The temperature does not change along the axial direction of the beam, but only exists on the cross section $T(x, y)$

(5) The horizontal displacement of the structure caused by torsion is not considered

(6) The warping function sum $\beta_{z}^{\prime}$ and $f_{z}^{\prime}$ are used for theoretical analysis, and the assumption of plane section in pure torsion theoretical analysis is not used

The coordinate system of the curved beam is shown in Figure 1.

2.2. The Solution of Vertical Deflection Torsion Angle and Warping Function. According to $[4,7]$, the geometric equations of vertical bending and torsion of curved beams are as follows:

$$
\begin{aligned}
k_{x} & =\frac{\mathrm{d}^{2} v}{\mathrm{~d} z^{2}}-\frac{\phi}{r} \\
& =\frac{\mathrm{d}^{2} v}{r^{2} \mathrm{~d} \varphi^{2}}-\frac{\phi}{r}, \\
\tau_{z}^{\prime} & =\frac{\mathrm{d} \tau}{\mathrm{d} z}=\frac{\mathrm{d} \phi}{\mathrm{d} z}+\frac{\mathrm{d} v}{r \mathrm{~d} z} \\
& =\frac{\mathrm{d} \phi}{r \mathrm{~d} \varphi}+\frac{\mathrm{d} v}{r^{2} \mathrm{~d} \varphi}, \\
f_{z}^{\prime} & =\frac{\mathrm{d} f}{\mathrm{~d} z} \\
& =\frac{\mathrm{d} \beta}{r \mathrm{~d} z}+\frac{\mathrm{d} v}{r \mathrm{~d} z} \\
& =\frac{\mathrm{d} \beta}{r \mathrm{~d} \varphi}+\frac{\mathrm{d} v}{r^{2} \mathrm{~d} \varphi},
\end{aligned}
$$

where $k_{x}$ is the vertical bending curvature; $\tau_{z}^{\prime}$ is the converted twist rate, which is the torsional angle per unit length; $f_{z}^{\prime}$ is the closed section warping function corresponding to $\tau_{z}^{\prime} ; v$ is the vertical deflection; $\phi$ is the section torsion angle; $\beta_{z}^{\prime}$ is the closed section warping function corresponding to $\phi_{z}^{\prime} ; \varphi$ is the center angle coordinate of the curved beam; and $r$ is the corresponding radius of the curved beam.

The physical equation is

$$
\begin{aligned}
M_{x} & =\mathrm{EI}_{x} k_{x}, \\
T_{s} & =\mathrm{GI}_{k} \tau_{z}^{\prime}, \\
T_{\omega} & =G\left(I_{\rho}-I_{k}\right)\left(\tau_{z}^{\prime}-f_{z}^{\prime}\right), \\
B & =-\mathrm{EI}_{\oplus} f^{\prime \prime},
\end{aligned}
$$

where $M_{x}$ is the bending moment in the axial direction; $T_{s}$ is the pure torsional torque; $T_{\omega}$ is the warping torsional torque; $B$ is the warping double moment; $I_{x}$ is the sectional bending moment of inertia in the $x$ axial direction; $I_{k}$ is the section pure torsion constant; $I_{\rho}$ is the polar moment of inertia of the section; and $I_{\varpi}$ is the moment of inertia of section warping. Total torque is $T=T_{s}+T_{\omega}$.

Considering a curved equal-section beam with center angle $\varphi_{0}$ and taking the midpoint of the circular arc as the coordinate zero point, determined by formulas (1)-(7), under the temperature effect, the potential energy of the structure is

$$
\begin{aligned}
W= & \frac{1}{2} \mathrm{EI}_{x} \int\left(k_{x}\right)^{2} r \mathrm{~d} \varphi+\frac{1}{2} G I_{k} \int_{-\left(\varphi_{0} / 2\right)}^{\left(\varphi_{0} / 2\right)}\left(\tau_{z}^{\prime}\right)^{2} r \mathrm{~d} \varphi+\frac{1}{2} G\left(I_{\rho}-I_{k}\right) \int_{-\left(\varphi_{0} / 2\right)}^{\left(\varphi_{0} / 2\right)}\left(\tau_{z}^{\prime}-f_{z}^{\prime}\right)^{2} r \mathrm{~d} \varphi+\frac{1}{2} \mathrm{EI}_{\oplus} \int_{-\left(\varphi_{0} / 2\right)}^{\left(\varphi_{0} / 2\right)}\left(f^{\prime \prime}\right)^{2} r \mathrm{~d} \varphi \\
& -E \int_{-\left(\varphi_{0} / 2\right)}^{\left(\varphi_{0} / 2\right)}\left[\iint \alpha T(x, y) \times\left(k_{x} y-f_{z z}^{\prime \prime} \omega\right) \mathrm{d} A\right] r \mathrm{~d} \varphi,
\end{aligned}
$$

where $\alpha$ is the coefficient of thermal expansion. The first four terms of the potential energy expression are strain energy, and the fifth term is temperature load potential energy. According to the principle of minimum potential energy, the possible deformation is the deformation that makes the potential energy reach the stationary value. For statically indeterminate simply supported curved beams, the function curves $v(\varphi), \tau(\varphi)$, and $f(\varphi)$ (or $v(\varphi), \phi(\varphi)$, and $\beta(\varphi)$ ) are needed to make the strain energy $W$ get the minimum value. Let $\delta W=0$ so that formulas (9)-(16) are obtained, where (9)-(11) are governing equations and (12)-(16) are boundary conditions. Get the governing equation: 


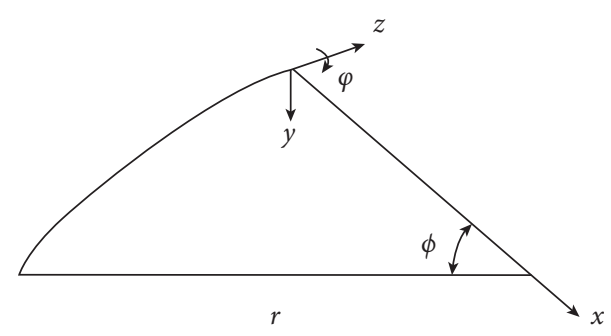

Figure 1: Curve girder coordinate system.

$$
\begin{aligned}
& \mathrm{EI}_{x}\left(\frac{\mathrm{d}^{4} v}{r^{2} \mathrm{~d} \varphi^{4}}+\frac{\mathrm{d}^{2} v}{r^{2} \mathrm{~d} \varphi^{2}}-\frac{\mathrm{d}^{2} \tau}{r \mathrm{~d} \varphi^{2}}\right)-\mathrm{GI}_{k} \frac{\mathrm{d}^{2} \tau}{r \mathrm{~d} \varphi^{2}}+\frac{\mathrm{EI}_{\oplus}}{r^{2}} \frac{\mathrm{d}^{4} f}{r \mathrm{~d} \varphi^{4}}=0, \\
& E\left[\iint \alpha T(x, y) y \mathrm{~d} A\right]-\mathrm{EI}_{x}\left(\frac{\mathrm{d}^{2} v}{r^{2} \mathrm{~d} \varphi^{2}}+\frac{v}{r^{2}}-\frac{\tau}{r}\right)-\mathrm{GI}_{k} \frac{\mathrm{d}^{2} \tau}{r \mathrm{~d} \varphi^{2}}-G\left(I_{\rho}-I_{k}\right)\left(\frac{\mathrm{d}^{2} \tau}{r \mathrm{~d} \varphi^{2}}-\frac{\mathrm{d}^{2} f}{r \mathrm{~d} \varphi^{2}}\right)=0, \\
& G\left(I_{\rho}-I_{k}\right)\left(\frac{\mathrm{d}^{2} \tau}{r \mathrm{~d} \varphi^{2}}-\frac{\mathrm{d}^{2} f}{r \mathrm{~d} \varphi^{2}}\right)+\frac{E I_{\varpi}}{r^{2}} \frac{\mathrm{d}^{4} f}{r \mathrm{~d} \varphi^{4}}=0 .
\end{aligned}
$$

Boundary conditions are

$$
\begin{aligned}
& {\left.\left[\mathrm{EI}_{x}\left(\frac{\mathrm{d}^{2} v}{r^{2} \mathrm{~d} \varphi^{2}}+\frac{v}{r^{2}}-\frac{\tau}{r}\right)+\frac{\mathrm{EI}_{\varpi}}{r^{2}} \frac{\mathrm{d}^{2} f}{r \mathrm{~d} \varphi^{2}}-E \iint \alpha T(x, y) y \mathrm{~d} A+\frac{E}{r} \iint \alpha T(x, y) \varpi \mathrm{d} A\right] \frac{\mathrm{d} \delta v}{r \mathrm{~d} \varphi}\right|_{-\left(\varphi_{0} / 2\right)} ^{\left(\varphi_{0} / 2\right)}=0,} \\
& {\left.\left[\mathrm{GI}_{k} \frac{\mathrm{d} \tau}{r \mathrm{~d} \varphi}-\frac{\mathrm{EI}_{\omega}}{r^{2}} \frac{\mathrm{d}^{4} f}{r \mathrm{~d} \varphi^{4}}-\mathrm{EI}_{x}\left(\frac{\mathrm{d}^{3} v}{r^{2} \mathrm{~d} \varphi^{3}}+\frac{\mathrm{d} v}{r^{2} \mathrm{~d} \varphi}-\frac{\mathrm{d} \tau}{r \mathrm{~d} \varphi}\right)\right] \frac{\delta v}{r}\right|_{-\left(\varphi_{0} / 2\right)} ^{\left(\varphi_{0} / 2\right)}=0,} \\
& {\left.\left[\mathrm{GI}_{k} \frac{\mathrm{d} \tau}{r \mathrm{~d} \varphi}+G\left(I_{\rho}-I_{k}\right)\left(\frac{\mathrm{d} \tau}{r \mathrm{~d} \varphi}-\frac{\mathrm{d} f}{r \mathrm{~d} \varphi}\right)\right] \delta \phi\right|_{-\left(\varphi_{0} / 2\right)} ^{\left(\varphi_{0} / 2\right)}=0} \\
& \left.\left(\frac{\mathrm{EI}_{\oplus}}{r} \frac{\mathrm{d}^{2} f}{r \mathrm{~d} \varphi^{2}}+E \iint \alpha T(x, y) \oplus \mathrm{d} A\right) \frac{\mathrm{d} \delta \beta}{r \mathrm{~d} \varphi}\right|_{-\left(\varphi_{0} / 2\right)} ^{\left(\varphi_{0} / 2\right)}=0, \\
& {\left.\left[-G\left(I_{\rho}-I_{k}\right)\left(\frac{\mathrm{d} \tau}{r \mathrm{~d} \varphi}-\frac{\mathrm{d} f}{r \mathrm{~d} \varphi}\right)-\frac{\mathrm{EI}_{\oplus}}{r^{2}} \frac{\mathrm{d}^{3} \tau}{r \mathrm{~d} \varphi^{3}}\right] \delta \beta\right|_{-\left(\varphi_{0} / 2\right)} ^{\left(\varphi_{0} / 2\right)}=0}
\end{aligned}
$$

At the bearing, which is $\varphi= \pm \varphi_{0} / 2$, because the bearing limits the torsion and vertical displacement, $\delta \phi=0, \delta v=0$, and $\delta \beta=0$. Boundary conditional expressions (13), (14), and (16) are naturally satisfied. However, simply supported bearings cannot limit the displacement $\mathrm{d} v / \mathrm{d} \varphi$ and $\mathrm{d} \beta / \mathrm{d} \varphi$, so $\mathrm{d} \delta v / r \mathrm{~d} \varphi$ and $\mathrm{d} \delta \beta / r \mathrm{~d} \varphi$ are not 0 in $\varphi= \pm \varphi_{0} / 2$, so in $\varphi= \pm \varphi_{0} / 2$,

$$
\begin{aligned}
& \mathrm{EI}_{x}\left(\frac{\mathrm{d}^{2} v}{r^{2} \mathrm{~d} \varphi^{2}}+\frac{v}{r^{2}}-\frac{\tau}{r}\right)+\frac{\mathrm{EI}_{\omega}}{r^{2}} \frac{\mathrm{d}^{2} f}{r \mathrm{~d} \varphi^{2}}-E \iint \alpha T(x, y) y \mathrm{~d} A+\frac{E}{r} \iint \alpha T(x, y) \oplus \mathrm{d} A=0, \\
& \frac{\mathrm{EI}_{\oplus}}{r} \frac{\mathrm{d}^{2} f}{r \mathrm{~d} \varphi^{2}}+E \iint \alpha T(x, y) \oplus \mathrm{d} A=0 .
\end{aligned}
$$


First, it is solved by governing equations (9)-(11) and obtained by (10):

$$
\mathrm{EI}_{x}\left(\frac{\mathrm{d}^{2} v}{r^{2} \mathrm{~d} \varphi^{2}}+\frac{v}{r^{2}}\right)=E\left[\iint \alpha T(x, y) y \mathrm{~d} A\right]+\mathrm{EI}_{x} \frac{\tau}{r}-\mathrm{GI}_{\rho} \frac{\mathrm{d}^{2} \tau}{r \mathrm{~d} \varphi^{2}}+G\left(I_{\rho}-I_{k}\right) \frac{\mathrm{d}^{2} f}{r \mathrm{~d} \varphi^{2}}
$$

Substitute equation (19) into equation (9) to get

$$
\left[G\left(I_{\rho}-I_{k}\right)+\frac{\mathrm{EI}_{\varpi}}{r^{2}}\right] \frac{\mathrm{d}^{4} f}{r \mathrm{~d} \varphi^{4}}=\mathrm{GI}_{\rho} \frac{\mathrm{d}^{4} \tau}{r \mathrm{~d} \varphi^{4}}+\mathrm{GI}_{k} \frac{\mathrm{d}^{2} \tau}{r \mathrm{~d} \varphi^{2}} .
$$

$$
\frac{\mathrm{d}^{2} \tau}{r \mathrm{~d} \varphi^{2}}=\frac{\mathrm{d}^{2} f}{r \mathrm{~d} \varphi^{2}}-\frac{\mathrm{EI}_{\varpi}}{r^{2} G\left(I_{\rho}-I_{k}\right)} \frac{\mathrm{d}^{4} f}{r \mathrm{~d} \varphi^{4}}
$$

From (11),

$$
-\frac{\mathrm{GI}_{\rho} \mathrm{EI}_{\varpi}}{r^{2}} \frac{\mathrm{d}^{6} f}{r \mathrm{~d} \varphi^{6}}+G\left(I_{\rho}-I_{k}\right) \mathrm{GI}_{k} \frac{\mathrm{d}^{2} f}{r \mathrm{~d} \varphi^{2}}+\left[\mathrm{GI}_{k} G\left(I_{\rho}-I_{k}\right)-\mathrm{GI}_{\rho} \frac{\mathrm{EI}_{\omega}}{r^{2}}\right] \frac{\mathrm{d}^{4} f}{r \mathrm{~d} \varphi^{4}}=0 .
$$

Solving it,

$$
\begin{aligned}
\frac{\mathrm{d}^{2} f}{\mathrm{~d} \varphi^{2}} & =C_{1} \cos \varphi+C_{2} \sin \varphi+C_{3} \cosh k \varphi+C_{4} \sinh k \varphi, \\
f & =-C_{1} \cos \varphi-C_{2} \sin \varphi+\frac{1}{k^{2}} C_{3} \cosh k \varphi+\frac{1}{k^{2}} C_{4} \sinh k \varphi+C_{5} \varphi+C_{6},
\end{aligned}
$$

and among them,

$$
k=\sqrt{\frac{r^{2} G\left(I_{\rho}-I_{k}\right) \mathrm{GI}_{k}}{\mathrm{EI}_{\omega} \mathrm{GI}_{\rho}}}
$$

Because the symmetric structure has symmetric stress and deformation under symmetric temperature load, $f$ is an even function. $C_{2}=0, C_{4}=0$, and $C_{5}=0$ can be known from symmetry.

Substitute (24) into (21) to get

$$
\frac{\mathrm{d}^{2} \tau}{\mathrm{d} \varphi^{2}}=\left[1+\frac{\mathrm{EI}_{\varpi}}{r^{2} G\left(I_{\rho}-I_{k}\right)}\right]\left(C_{1} \cos \varphi+C_{2} \sin \varphi\right)+\left(1-\frac{I_{k}}{I_{\rho}}\right)\left(C_{3} \cosh k \varphi+C_{4} \sinh k \varphi\right) .
$$

Solving it,

$$
\tau=-\left[1+\frac{\mathrm{EI}_{\oplus}}{r^{2} G\left(I_{\rho}-I_{k}\right)}\right]\left(C_{1} \cos \varphi+C_{2} \sin \varphi\right)+\frac{1}{k^{2}}\left(1-\frac{I_{k}}{I_{\rho}}\right)\left(C_{3} \cosh k \varphi+C_{4} \sinh k \varphi\right)+C_{7} \varphi+C_{8} .
$$

Similarly, $\tau$ is an even function, and Substitute equations (24) and (27) into equation (19), sort $C_{2}=0, C_{4}=0$, and $C_{7}=0$ are known by symmetry. out, and solve them.

$$
\begin{aligned}
\frac{v}{r}= & C_{9} \cos \varphi+C_{10} \sin \varphi+\left\{\frac{G\left(I_{\rho}-I_{k}\right)}{\mathrm{EI}_{x}}-\left(\frac{\mathrm{GI}_{\rho}}{\mathrm{EI}_{x}}+1\right)\left[1+\frac{\mathrm{EI}_{\varpi}}{r^{2} G\left(I_{\rho}-I_{k}\right)}\right]\right\} \times\left(\frac{1}{2} C_{1} \varphi \sin \varphi-\frac{1}{2} C_{2} \varphi \cos \varphi\right) \\
& +\frac{1}{k^{2}\left(1+k^{2}\right)}\left(1-\frac{I_{k}}{I_{\rho}}\right)\left(C_{3} \cosh k \varphi+C_{4} \sinh k \varphi\right)+\frac{r \iint \alpha T(x, y) y \mathrm{~d} A}{I_{x}}+C_{7} \varphi+C_{8} .
\end{aligned}
$$


In the same way, $v$ is an even function, and $C_{2}=0, C_{4}=$ $0, C_{5}=0$, and $C_{10}=0$ are known by symmetry, obtained by sorting out (24), (26), (27), and (28).

$$
\begin{aligned}
f= & -C_{1} \cos \varphi+\frac{1}{k^{2}} C_{3} \cosh k \varphi+C_{6}, \\
\tau= & -\left[1+\frac{\mathrm{EI}_{\oplus}}{r^{2} G\left(I_{\rho}-I_{k}\right)}\right] C_{1} \cos \varphi+\frac{1}{k^{2}}\left(1-\frac{I_{k}}{I_{\rho}}\right) C_{3} \cosh k \varphi+C_{8}, \\
\frac{v}{r}= & C_{9} \cos \varphi+\frac{r \iint \alpha T(x, y) y \mathrm{~d} A}{I_{x}}+C_{8}+\frac{1}{2} C_{1} \varphi \sin \varphi\left\{\frac{G\left(I_{\rho}-I_{k}\right)}{\mathrm{EI}_{x}}-\left(\frac{\mathrm{GI}_{\rho}}{\mathrm{EI}_{x}}+1\right)\left[1+\frac{\mathrm{EI}_{\varrho}}{r^{2} G\left(I_{\rho}-I_{k}\right)}\right]\right\} \\
& +\frac{1}{k^{2}\left(1+k^{2}\right)}\left(1-\frac{I_{k}}{I_{\rho}}\right) C_{3} \cosh k \varphi .
\end{aligned}
$$

Considering boundary conditions (17) and (18), substituting equations (18), (30), and (31) into equation (17), we get

$$
-\mathrm{EI}_{x}\left[\frac{\mathrm{GI}_{k}}{\mathrm{EI}_{x}}+\frac{I_{\omega} I_{\rho}}{r^{2}\left(I_{\rho}-I_{k}\right) I_{x}}\right] C_{1} \cos \frac{\varphi_{0}}{2}=0 .
$$

For most sections, there are

$$
\frac{\mathrm{GI}_{k}}{\mathrm{EI}_{x}}+\frac{I_{\omega} I_{\rho}}{r^{2}\left(I_{\rho}-I_{k}\right) I_{x}} \neq 0
$$

Therefore, substitute equation (29) into equation (18), and get $C_{1}=0$.

$$
C_{3}=\frac{r^{2} \iint \alpha T(x, y) \omega \mathrm{d} A}{I_{\varpi} \cosh k\left(\varphi_{0} / 2\right)}
$$

By the boundary conditions of the structure,

$$
\begin{gathered}
v\left(-\frac{\varphi_{0}}{2}\right)=0, \\
v\left(\frac{\varphi_{0}}{2}\right)=0, \\
\phi\left(-\frac{\varphi_{0}}{2}\right)=0, \\
\phi\left(\frac{\varphi_{0}}{2}\right)=0, \\
\tau\left(-\frac{\varphi_{0}}{2}\right)=0 \\
\tau\left(\frac{\varphi_{0}}{2}\right)=0 .
\end{gathered}
$$

From equations (30), (34), and (35), we get

$$
C_{8}=-\frac{1}{k^{2}}\left(1-\frac{I_{k}}{I_{\rho}}\right) \frac{r^{2} \iint \alpha T(x, y) \omega \mathrm{d} A}{I_{\varpi}} .
$$

So,

$$
\tau=\frac{1}{k^{2}}\left(1-\frac{I_{k}}{I_{\rho}}\right) \frac{r^{2} \iint \alpha T(x, y) \omega \mathrm{d} A}{I_{\varpi}} \times\left[\frac{\cosh k \varphi}{\cosh k\left(\varphi_{0} / 2\right)}-1\right] .
$$

It is obtained from formulas (30), (34), (35), and (36) that 
Therefore, the solution of vertical deflection $v$, section torsion angle $\phi$, and warping function $f_{z}^{\prime}$ is

$$
\begin{aligned}
\frac{v}{r} & =\left(1-\frac{I_{k}}{I_{\rho}}\right) \frac{r^{2} \iint \alpha T(x, y) \omega \mathrm{d} A}{I_{\varpi}} \times\left[\frac{1}{1+k^{2}} \frac{\cos \varphi}{\cos \left(\varphi_{0} / 2\right)}+\frac{1}{k^{2}} \frac{1}{1+k^{2}} \frac{\cosh k \varphi}{\cosh k\left(\varphi_{0} / 2\right)}-\frac{1}{k^{2}}\right]+\frac{r \iint \alpha T(x, y) y \mathrm{~d} A}{I_{x}}\left(1-\frac{\cos \varphi}{\cos \left(\varphi_{0} / 2\right)}\right), \\
\phi & =\tau-\frac{v}{r}=\frac{1}{1+k^{2}}\left(1-\frac{I_{k}}{I_{\rho}}\right) \frac{r^{2} \iint \alpha T(x, y) \omega \mathrm{d} A}{I_{\varpi}} \times\left[\frac{\cosh k \varphi}{\cosh k\left(\varphi_{0} / 2\right)}-\frac{\cos \varphi}{\cos \left(\varphi_{0} / 2\right)}\right]+\frac{r \iint \alpha T(x, y) y \mathrm{~d} A}{I_{x}}\left(\frac{\cos \varphi}{\cos \left(\varphi_{0} / 2\right)}-1\right), \\
\frac{\mathrm{d} f}{\mathrm{~d} z} & =\frac{\mathrm{d} f}{r \mathrm{~d} \varphi} \\
& =\frac{r \iint \alpha T(x, y) \omega \mathrm{d} A}{k I_{\oplus} \cosh k\left(\varphi_{0} / 2\right)} \sinh k \varphi .
\end{aligned}
$$

If the warping effect in torsion is ignored, the potential energy of the structure is

$$
W=\frac{1}{2} \mathrm{EI}_{x} \int_{-\left(\varphi_{0} / 2\right)}^{\left(\varphi_{0} / 2\right)}\left(k_{x}\right)^{2} r \mathrm{~d} \varphi+\frac{1}{2} \mathrm{GI}_{k} \int_{-\left(\varphi_{0} / 2\right)}^{\left(\varphi_{0} / 2\right)}\left(\tau_{z}^{\prime}\right)^{2} r \mathrm{~d} \varphi-E \int_{-\left(\varphi_{0} / 2\right)}^{\left(\varphi_{0} / 2\right)}\left[\iint \alpha T(x, y) \times k_{x} y \mathrm{~d} A\right] r \mathrm{~d} \varphi
$$

It can be obtained by a similar calculation process as above.

$$
\begin{aligned}
& \frac{v}{r}=\frac{r \iint \alpha T(x, y) y \mathrm{~d} A}{I}\left[1-\frac{\cos \varphi}{\cos \left(\varphi_{0} / 2\right)}\right], \\
& \phi=\frac{r \iint \alpha T(x, y) y \mathrm{~d} A}{I}\left[\frac{\cos \varphi}{\cos \left(\varphi_{0} / 2\right)}-1\right] .
\end{aligned}
$$

Because the coordinates on the symmetric box section are left and right antisymmetric about the section, for the temperature distribution of the left and right symmetric section, such as the temperature gradient along the beam height specified in General Code for Design of Highway Bridges and Culverts (JTG D60-2015)[23], there are.

$$
\iint \alpha T(x, y) \omega \mathrm{d} A=0 .
$$

At this time, equations (41) and (42) are completely consistent with equations (45) and (46). Therefore, under the assumptions mentioned above, the deformation calculation results of the thin-walled closed curve box girder with and without considering the warping effect are the same for the temperature distribution of the left and right sections. On the contrary, the deformation calculation results of the thinwalled closed curve box girder with and without the warping effect are different for the temperature distribution of the left-right asymmetric section. It can be seen that, for the thin-walled closed curve box girder, due to the existence of the warping effect, the transverse temperature gradient distribution in the plane will affect the vertical deflection, torsion angle, and warping out of plane. This property is different from that of the straight beam, which shows that the transverse temperature gradient is also of great significance when considering the temperature field distribution and temperature effect of the curved beam.

\section{Finite Element Verification}

The thin-walled closed curved box girder is taken as an example, and the sectional shape is shown in Figure 2. The temperature load here is the temperature gradient load specified in 《Code for Design of Concrete Structures of Railway Bridges and Culverts》(TB 10092-2017) [24], $T(y)=20 \mathrm{e}^{-5 y}$ and $T(x)=16 \mathrm{e}^{-7 x}$, whose unit is ${ }^{\circ} \mathrm{C}$. Section characteristics and data related to calculation are shown in Table 1.

3.1. Single-Span Statically Indeterminate Simply Supported Curved Beam. Solid45 solid element in finite element analysis software Ansys is used to establish a single-span curved beam model, and the boundary conditions are set with reference to the bearing conditions of the structure, as shown in Figure 3. The deflection calculation result diagram is shown in Figure 4, and the calculation results of the finite element and theoretically calculated deflection $v$ at some coordinate angles are shown in Table 2 and Figure 5. The relative difference in Table 2 is the comparison result between the theoretical value and finite element calculation result (average value). 


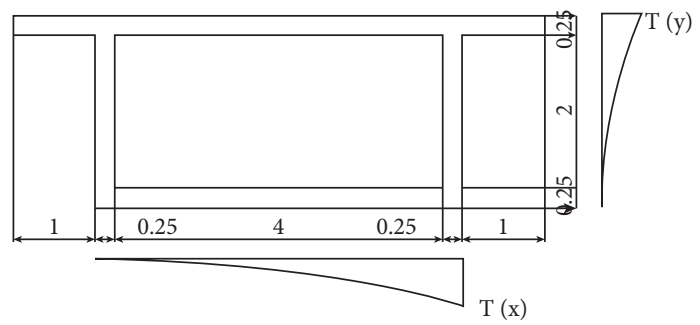

Figure 2: Cross section and temperature load (unit: $\mathrm{m}$ ).

TABle 1: Section geometry characteristics and related data.

\begin{tabular}{lcccccc}
\hline$A\left(\mathrm{~m}^{2}\right)$ & Height of the shape center $(\mathrm{m})$ & Height of the shear center $(\mathrm{m})$ & $I_{x}\left(\mathrm{~m}^{4}\right)$ & $I_{y}\left(\mathrm{~m}^{4}\right)$ & $I_{k}\left(\mathrm{~m}^{4}\right)$ & $I_{\rho}\left(\mathrm{m}^{4}\right)$ \\
\hline 3.75 & 1.40 & 1.416 & 3.74 & 12.14 & 7.03 \\
\hline$I_{w}\left(\mathrm{~m}^{6}\right)$ & $\alpha$ & $E(\mathrm{GPa})$ & Poisson's ratio $v$ & $r(\mathrm{~m})$ & Length per span $l(\mathrm{~m})$ & $\varphi_{0}(\mathrm{rad})$ \\
\hline 1.04 & $1 * 10^{-5}$ & 34.5 & 0.2 & 100 & 20 & 0.2 \\
\hline
\end{tabular}

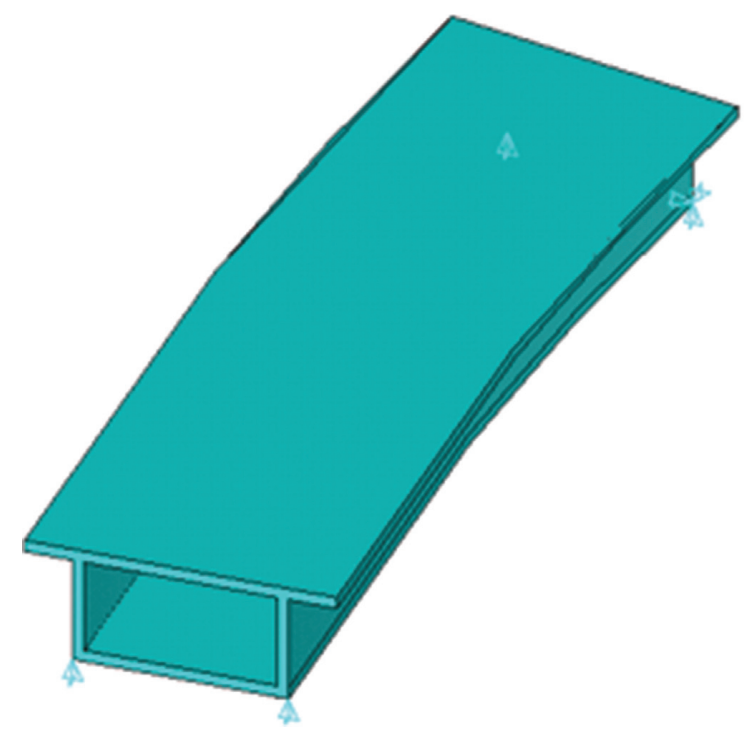

FIGURE 3: Single-span curved beam finite element model.
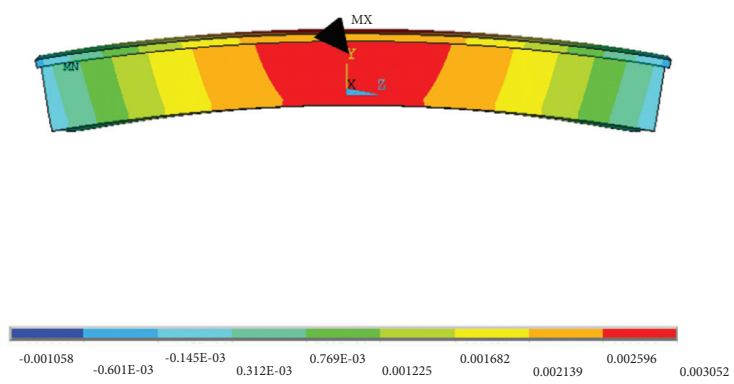

FIGURE 4: Finite element calculation results for the single-span curved beam.

It can be seen from Table 2 that when calculating the vertical deflection of a single-span statically indeterminate simply supported curved beam, the calculated results with and without warpage are smaller than those calculated by the finite element method, the difference is about $5 \%$, and the results with warpage are slightly more accurate than those without warpage. 
TABLE 2: Comparison of deflection calculation results.

\begin{tabular}{lccccc}
\hline $\begin{array}{l}\text { Corresponding } \\
\text { coordinate angle }(\mathrm{rad})\end{array}$ & $\begin{array}{c}\text { Finite element } \\
\text { calculation result } \\
(\mathrm{mm})\end{array}$ & $\begin{array}{c}\text { Considering } \\
\text { warpage }(\mathrm{mm})\end{array}$ & $\begin{array}{c}\text { Relative difference } \\
\text { considering warping } \\
(\%)\end{array}$ & $\begin{array}{c}\text { Ignoring } \\
\text { warpage }(\mathrm{mm})\end{array}$ & $\begin{array}{c}\text { Relative difference when } \\
\text { warpage is ignored (\%) }\end{array}$ \\
\hline-0.08 & 1.06 & 1.00 & -5.66 & 1.00 & -5.66 \\
-0.06 & 1.87 & 1.78 & -4.81 & 1.77 & -5.35 \\
-0.04 & 2.46 & 2.34 & -4.88 & 2.32 & -5.69 \\
-0.02 & 2.81 & 2.67 & -4.98 & 2.66 & -5.34 \\
0.00 & 2.93 & 2.78 & -5.12 & 2.66 & -5.67 \\
0.02 & 2.82 & 2.67 & -5.32 & 2.32 & -5.69 \\
0.04 & 2.46 & 2.34 & -5.32 & 1.77 & -5.85 \\
0.06 & 1.88 & 1.78 & -5.66 & 1.00 & -5.66 \\
0.08 & 1.06 & 1.00 & & \\
\hline
\end{tabular}

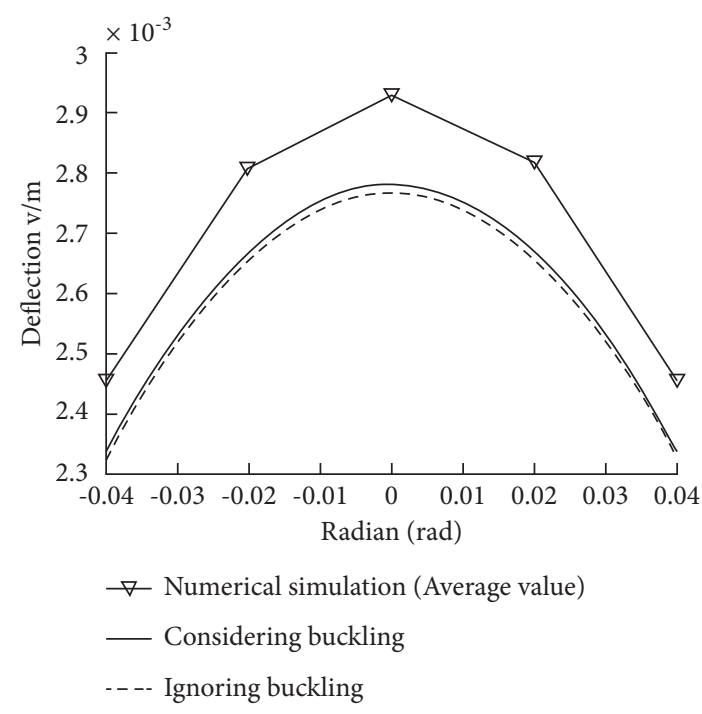

Figure 5: Comparison of deflection calculation results.

3.2. Multispan Continuous Curved Beam. Taking the deformation equation of a single-span statically indeterminate simply supported curved beam under temperature effect as the basic structure, the multispan continuous curved beam is solved according to the force method equation. In this structure, the generalized forces are the bending moment at the intersection point of each span and the warping double moments. Generalized displacement is the relative rotation angle and warpage at the intersection point, and the magnitudes are, respectively,

$$
\begin{aligned}
\delta & =\frac{\mathrm{d} v}{\mathrm{~d} z} \\
& =\frac{\mathrm{d} v}{r \mathrm{~d} \varphi}, \\
\mu & =\frac{\mathrm{d} f}{\mathrm{~d} z} \\
& =\frac{\mathrm{d} f}{r \mathrm{~d} \varphi} .
\end{aligned}
$$

Therefore, under the action of temperature load,

$$
\begin{aligned}
& \delta_{1 T}=\delta_{2 T}=\cdots=\delta_{i T} \\
& =\left.2 \frac{\mathrm{d} v}{r \mathrm{~d} \varphi}\right|_{\varphi=\left(\varphi_{0} / 2\right)}=2 \frac{r \iint \alpha T(x, y) y \mathrm{~d} A}{I_{x}} \tan \frac{\varphi_{0}}{2}+2\left(1-\frac{I_{k}}{I_{\rho}}\right) \frac{r^{2} \iint \alpha T(x, y) \omega \mathrm{d} A}{I_{\varpi}} \times\left(-\frac{1}{1+k^{2}} \tan \frac{\varphi_{0}}{2}+\frac{1}{k^{2}} \frac{1}{1+k^{2}} \tanh k \frac{\varphi_{0}}{2}\right) \\
& \mu_{1 T}=\mu_{2 T}=\cdots=\mu_{i T} \\
& =\left.2 \frac{\mathrm{d} f}{r \mathrm{~d} \varphi}\right|_{\varphi=\left(\varphi_{0} / 2\right)}=2 \frac{r \iint \alpha T(x, y) \bowtie \mathrm{d} A}{k I_{\varpi}} \tanh k \frac{\varphi_{0}}{2} . \\
& {\left[\boldsymbol{\delta}_{\mathbf{i j}}^{\mathbf{M}}\right]\left(M_{i}\right)+\left[\boldsymbol{\delta}_{\mathbf{i j}}^{\mathbf{B}}\right]\left(B_{i}\right)+\left(\delta_{i T}\right)=(0),} \\
& {\left[\boldsymbol{\mu}_{\mathbf{i j}}^{\mathbf{M}}\right]\left(M_{i}\right)+\left[\boldsymbol{\mu}_{\mathbf{i j}}^{\mathbf{B}}\right]\left(B_{i}\right)+\left(\mu_{i T}\right)=(0),}
\end{aligned}
$$
$2 r \iint \alpha T(x, y) y \mathrm{~d} A / I_{x} \tan \varphi_{0} / 2$.

And the force method equation of the $n+1$ span continuous curved beam is where $\left[\delta_{\mathbf{i j}}^{\mathbf{M}}\right],\left[\delta_{\mathbf{i j}}^{\mathbf{B}}\right],\left[\mu_{\mathbf{i j}}^{\mathbf{M}}\right]$, and $\left[\mu_{\mathbf{i j}}^{\mathbf{B}}\right]$ are $n \times n$ order stiffness matrices, and each item in the matrix is given by [4]. 
TABLE 3: Comparison of the torque reaction.

\begin{tabular}{lcccccc}
\hline Bearing & $\begin{array}{c}\text { Bearing } \\
\text { torque }\end{array}$ & $\begin{array}{c}\text { Finite element } \\
\text { results }(\mathrm{kN} \cdot \mathrm{m})\end{array}$ & $\begin{array}{c}\text { Ignore warpage } \\
(\mathrm{kN} \cdot \mathrm{m})\end{array}$ & $\begin{array}{c}\text { Relative } \\
\text { difference }(\%)\end{array}$ & $\begin{array}{c}\text { Consider } \\
\text { warpage }(\mathrm{kN} \cdot \mathrm{m})\end{array}$ & $\begin{array}{c}\text { Relative } \\
\text { difference }(\%)\end{array}$ \\
\hline \multirow{2}{*}{ Bearings near the midspan } & $T_{2}$ & 1224.05 & 1388.32 & 13.42 & 1310.92 & 7.10 \\
& $T_{3}$ & 1254.59 & 295.47 & \multirow{2}{*}{278.11} & -9.64 & \multirow{2}{*}{310.44} \\
\hline Stay away from the bearings in & $T_{1}$ & 309.70 & -13.79 & 5.07 \\
the middle of the span & $T_{4}$ & & & 0.24 \\
\hline
\end{tabular}

For three-span continuous curved beams, the corresponding parameters are the same as those in Figure 2 and Table 1. Under the temperature load specified in Figure 2, the calculation results of the bearing torque reaction of the statically indeterminate structure are shown in Table 3 , and the negative sign indicating direction is omitted from the calculation results of torque in the table.

A three-span continuous beam model is established with Solid 45 solid element in finite element analysis software Ansys, and the boundary conditions are arranged with reference to the bearing conditions of the structure, as shown in Figure 6. Under the temperature gradient load specified in Figure 2, the calculation results of finite element deflection and bearing torque reaction are shown in Figure 7, and the comparison between theoretical calculation results and finite element calculation results of each bearing torque reaction is shown in Table 3.

Comparing the calculation results of the reaction force of each support of the statically indeterminate structure, it is found that the relative difference between the calculation results considering warpage and the finite element calculation results is about $5 \%$, and the calculation results have sufficient accuracy, while the calculation results ignoring warpage have larger errors, and some items exceed $10 \%$. There is a big difference between the calculation results considering warpage and ignoring warpage, and the difference between each redundant force and bearing torque reaction force exceeds $5 \%$.

\section{Parameter Analysis}

The radius $r$, cross-section bottom length $l_{1}$, height $l_{2}$, and flange length $l_{3}$ (for example, the cross section shown in Figure 2 is $l_{1}=4 \mathrm{~m}, l_{2}=2 \mathrm{~m}$, and $l_{3}=1 \mathrm{~m}$ ) are taken as parameters for parameter analysis. The theoretical calculation results of the three-span continuous curved beam with different parameters are compared with the finite element calculation results. For the convenience of analysis, the bearing torque reaction near the middle of the span is taken as the comparison item.

4.1. Radius $r$. On the premise of keeping the cross-section shape, temperature gradient distribution, and other parameters unchanged, three results and relative differences of the bearing torque reaction near the midspan with radius $r$ of $60 \mathrm{~m}, 80 \mathrm{~m}, 100 \mathrm{~m}, 20 \mathrm{~m}$, and $140 \mathrm{~m}$ are obtained, as shown in Figure 8. It can be seen from Figure 8 that, with the decrease of radius, the bearing reaction force increases.

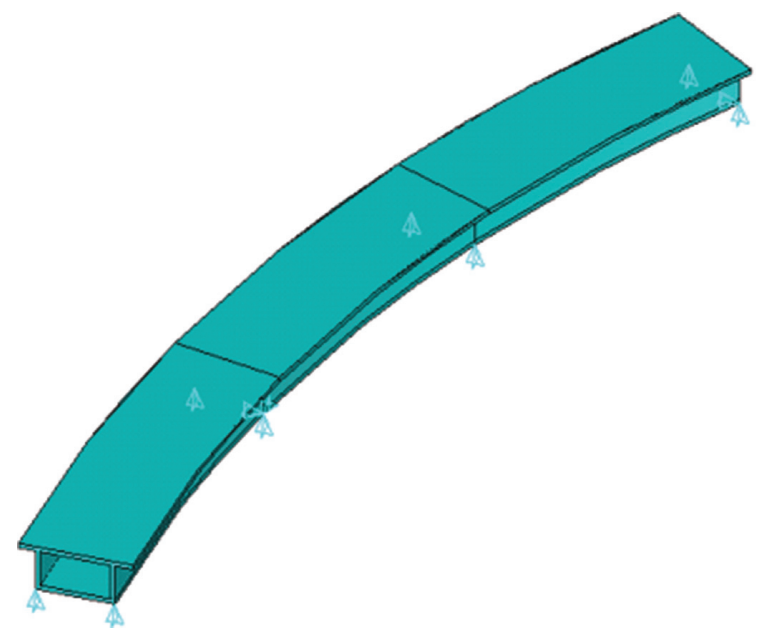

Figure 6: Multispan curved beam finite element model.
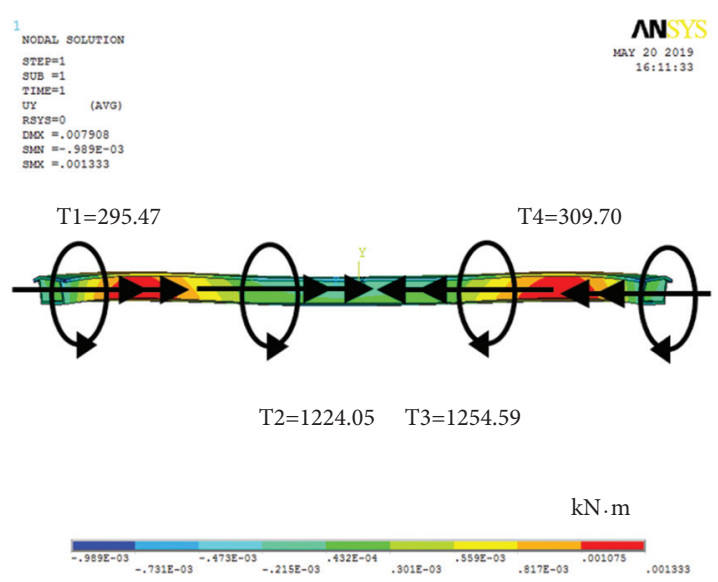

FIgURE 7: Finite element calculation results for the multispan curved beam.

However, when the radius is different, the relative difference between the calculated results of the torque reaction of the three bearings is basically unchanged. The relative difference between the calculation results considering warpage and ignoring warpage is $-5.5 \%$, and that between the calculation results considering warpage and the finite element calculation results is $5.8 \%$. It can be seen that the radius has little influence on the relative difference among the three calculation results. For other sections with different sizes and shapes, the same rules are also observed. Therefore, after parameter analysis, the radius $r=100 \mathrm{~m}$ is set. 

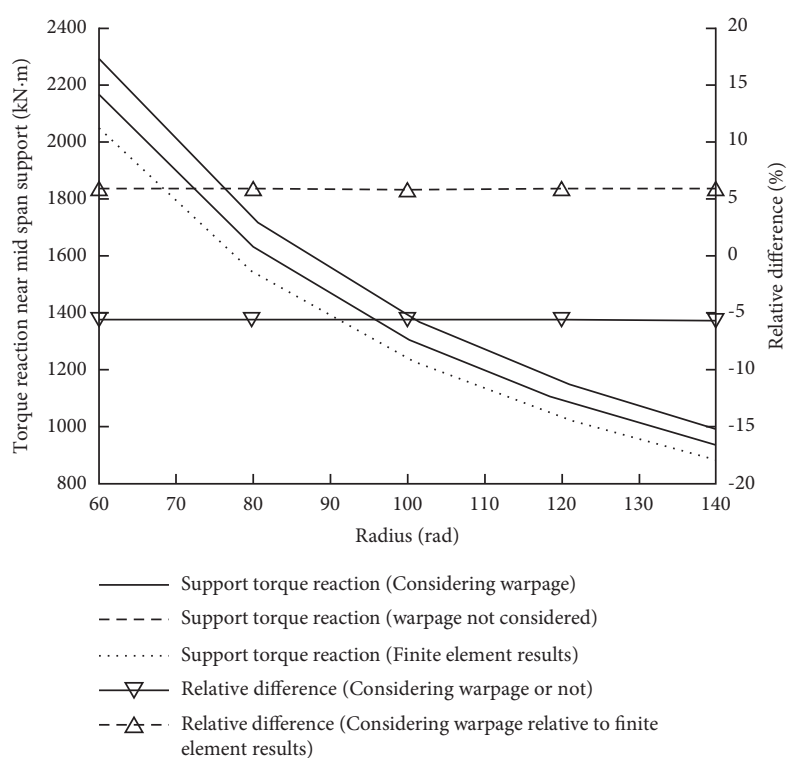

FIgURE 8: Torque reaction and relative difference with different radii.

TABLE 4: Torque reaction forces near the midspan support under different cross-section shapes.

\begin{tabular}{|c|c|c|c|c|c|c|c|c|c|c|c|c|}
\hline & \multicolumn{4}{|c|}{ Considering warpage $(\mathrm{kN} \cdot \mathrm{m})$} & \multicolumn{4}{|c|}{ Ignoring warpage $(\mathrm{kN} \cdot \mathrm{m})$} & \multicolumn{4}{|c|}{ Finite element result $(\mathrm{kN} \cdot \mathrm{m})$} \\
\hline Sectiona & $l_{1}=3 \mathrm{~m}$ & $l_{1}=4 \mathrm{~m}$ & $l_{1}=5 \mathrm{~m}$ & $l_{1}=6 \mathrm{~m}$ & $l_{1}=3 \mathrm{~m}$ & $l_{1}=4 \mathrm{~m}$ & $l_{1}=5 \mathrm{~m}$ & $l_{1}=6 \mathrm{~m}$ & $l_{1}=3 \mathrm{~m}$ & $l_{1}=4 \mathrm{~m}$ & $l_{1}=5 \mathrm{~m}$ & $l_{1}=6 \mathrm{~m}$ \\
\hline$l_{2}=1 \mathrm{~m}$ & 600 & 738 & 812 & 961 & 586 & 714 & 843 & 971 & 651 & 749 & 862 & 918 \\
\hline$l_{2}=1.5 \mathrm{~m}$ & 818 & 991 & 1203 & 1400 & 830 & 1006 & 1182 & 1358 & 769 & 979 & 1235 & 1388 \\
\hline$l_{2}=2 \mathrm{~m}$ & 907 & 1311 & 1593 & 1730 & 1079 & 1388 & 1525 & 1749 & 886 & 1285 & 1576 & 1651 \\
\hline$l_{2}=2.5 \mathrm{~m}$ & 1475 & 1655 & 1898 & 2082 & 1331 & 1601 & 1872 & 2143 & 1499 & 1696 & 1998 & 1999 \\
\hline$l_{2}=3 \mathrm{~m}$ & 1520 & 1857 & 2190 & 1980 & 1587 & 1903 & 2221 & 2540 & 1529 & 1790 & 2137 & 2061 \\
\hline
\end{tabular}

4.2. Cross-Section Bottom Edge Length $l_{1}$ and Height $l_{2}$. On the premise of keeping the temperature gradient distribution and radius unchanged, three results and relative differences of the bearing torque reaction near the midspan are obtained when the length $l_{1}$ of the cross-section base is $3 \mathrm{~m}, 4 \mathrm{~m}, 5 \mathrm{~m}$, and $6 \mathrm{~m}$ and the height $l_{2}$ is $1 \mathrm{~m}, 1.5 \mathrm{~m}, 2 \mathrm{~m}$, $2.5 \mathrm{~m}$, and $3 \mathrm{~m}$, as shown in Table 4 . See Figure 9 for the relationship between the relative difference between considering warpage, ignoring warpage, and finite element results and the warping torsion attenuation coefficient $\left(\sqrt{\left(1-I_{k} / I_{\rho}\right) \mathrm{GI}_{k} / \mathrm{EI}_{\emptyset}}\right)$, which is an important index to measure the warping torsion geometric characteristics of thin-walled curved beams, so this coefficient is used for analysis. It can be seen from Table 4 and Figure 9 that when the length $l_{1}$ and height $l_{2}$ of the bottom edge of the cross section change, the relative difference between the calculated result considering the warping effect and the finite element calculation result is about $5 \%$, which has good accuracy. Under the same parameters, the relative difference between the calculated result without considering warping and the finite element calculation result is higher than the former, and the relative difference of multiple calculation results is over $10 \%$ and sometimes reaches $22 \%$. However, there is no obvious positive or negative correlation between the two relative differences and the warping torsional attenuation coefficient.

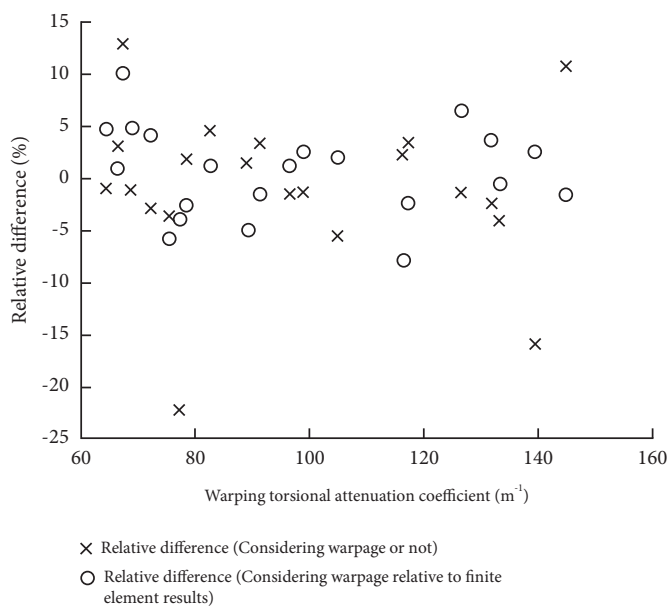

FIGURE 9: Relative difference of the torque reaction with different $l_{1}$ and $l_{2}$.

4.3. Flange Length $l_{3}$. On the premise of keeping temperature gradient distribution, radius, and other parameters unchanged, taking the three-span curved beam structure with cross sections of $l_{1}=4 \mathrm{~m}, l_{2}=2 \mathrm{~m}$, and flange length $l_{3}$ of 0.5 , $0.75,1,1.25$, and $1.5 \mathrm{~m}$ as an example, three results and relative differences of the bearing torque reaction near the midspan are calculated, which are considered warping, not 


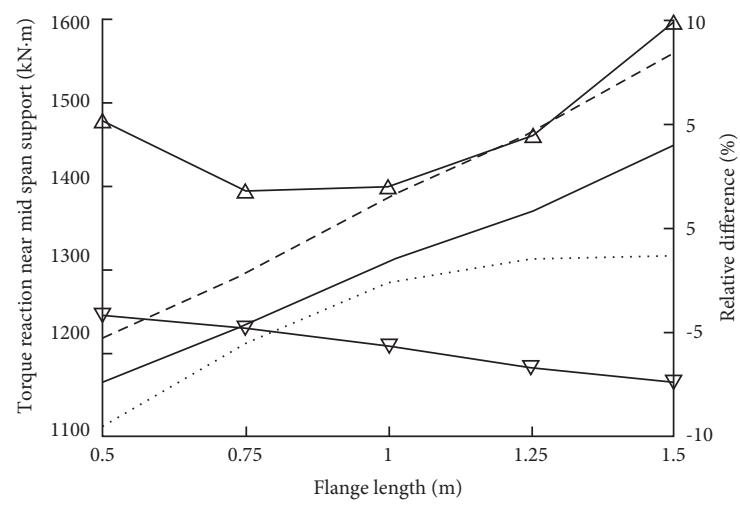

$$
\begin{aligned}
& - \text { Support torque reaction (Considering warpage) } \\
& \ldots--- \text { Support torque reaction (warpage not considered) } \\
& \ldots \ldots \ldots \text { Support torque reaction (Finite element results) } \\
& \square \quad \text { Relative difference (Considering warpage or not) } \\
& \square \quad \begin{array}{l}
\text { Relative difference (Considering warpage relative to finite } \\
\text { element results) }
\end{array}
\end{aligned}
$$

Figure 10: Torque reaction and relative difference with different flange lengths.

considered warping, and finite element calculation, as shown in Figure 10. It can be seen from Figure 10 that when the flange length $l_{3}$ is changed, the relative difference between the calculation result considering the warping effect and the finite element calculation result is between $2 \%$ and $10 \%$, which is larger than the previous analysis. When the flange is long, the theoretical solution proposed in this paper is not accurate enough. The reason may be that the shear lag effect is not considered, and the deformation assumption in this paper is inconsistent with the real deformation distribution of the flange. It is necessary to apply a more precise deformation assumption model to consider the calculation of the temperature effect of the curved beam structure in this case.

\section{Conclusion}

According to the basic differential equation of the curved beam and the principle of minimum energy, this study takes the primary torsional statically indeterminate simply supported curved beam as the basic structure and, according to whether the warping effect of the thin-walled closed curved box girder is considered, puts forward a simple analytical calculation method for the temperature deformation of the plane curved beam. Comparing the analytical calculation results with the finite element calculation results, the following conclusions are obtained:

(1) When calculating the vertical deflection of a singlespan statically indeterminate simply supported curved beam, the difference between the calculation results provided by this study with and without warping is small, both of which are about $5 \%$, and the calculation results with warping are slightly more accurate than those without warping. For multispan continuous curved beams, the relative difference between analytical calculation results and finite element calculation results considering warping is about $5 \%$ in the analysis, and the calculation results have sufficient accuracy. There is a big error in the calculation results when the warpage is ignored, and the relative difference of multiple calculation results is over $10 \%$. Therefore, considering the warping effect is helpful to calculate the temperature effect of the multispan continuous curved beam more accurately.

(2) By analyzing the parameters of curved beams with different cross sections and different radii, it is found that the relative difference between the theoretical calculation results and the finite element results considering the warping effect is greatly influenced by the cross-section shape, but less influenced by the radius size. However, there is no obvious positive or negative correlation between the relative difference and the warping torsional attenuation coefficient of the thin-walled box section.

(3) When the flange length is taken as the parameter, the relative difference between the calculation result considering the warping effect and the finite element calculation result reaches $10 \%$ when the flange is long. Therefore, more detailed deformation assumptions, such as shear lag deformation assumptions, are needed to more accurately consider the temperature effect calculation of the thin-walled curved box girder when the flange is long.

(4) Through theoretical calculation, it is found that, for the thin-walled closed curved box girder, the transverse temperature gradient distribution in the plane will affect the vertical deflection, torsion angle, and warpage out of plane. This property is different from that of the straight beam.

\section{Data Availability}

The data that support the findings of this study are available from the corresponding author upon reasonable request.

\section{Conflicts of Interest}

The authors declare that they have no conflicts of interest.

\section{Acknowledgments}

This work was supported by the Shandong Transportation Science and Technology Program (2013A08-03 and 2021B85).

\section{References}

[1] G. Zhang, R. Alberdi, and K. Khandelwal, "Analysis of threedimensional curved beams using isogeometric approach," Engineering Structures, vol. 117, no. 15, pp. 560-574, 2016.

[2] A. T. Luu, N. I. Kim, and J. Lee, "Isogeometric vibration analysis of free-form Timoshenko curved beams," Meccanica, vol. 50, no. 1, pp. 169-187, 2015.

[3] S. Rajasekaran, "Analysis of curved beams using a new differential transformation based curved beam element," Meccanica, vol. 49, no. 4, pp. 863-886, 2014. 
[4] J. Huang, Torsion Analysis of Thin-Walled Structures: Curved Beams and Oblique Supported Box beams, China Railway Publishing House, Beijing, China, 1998.

[5] Y. Shen, T. Song, and L. I. Guoping, "Torsion-resistant design in curved box-girder bridges with external tendons," Journal of Tongji University, vol. 43, no. 12, pp. 1777-1783, 2015.

[6] C. Wei and Q. Zeng, "Spatial analysis of thin-walled curved box girder considering warping, distortion and shear lag effect," China Civil Engineering Journal, vol. 33, no. 6, pp. 81-87, 2000.

[7] Y. Zhang and L. I. Qiao, "Analysis of flexural torsion of thinwalled curved box girder considering shear lag and confined torsional secondary shear deformation," China Civil Engineering Journal, vol. 42, no. 3, pp. 93-98, 2009.

[8] M. Zhou, L. I. Liyuan, and Y. Zhang, "Analysis of bending and torsion of curved box girder considering section shear deformation," Journal of Lanzhou Jiaotong University, vol. 6, no. 1, pp. 17-22, 2017.

[9] Y. Gan and G. Zhou, "Double warping displacement function method for static analysis of curved rectangular box girder," China Civil Engineering Journal, vol. 3, no. 4, pp. 63-69, 2010.

[10] Y. Gan, G. Zhou, and Y. Wu, "Energy variational method for lateral static analysis of curved thin-walled box girders," Chinese Journal of Solid Mechanics, vol. 32, no. 4, pp. 411-418, 2011.

[11] J. H. Lee and I. Kalkan, "Analysis of thermal environmental effects on precast, prestressed concrete bridge girders: temperature differentials and thermal deformations," Advances in Structural Engineering, vol. 15, no. 3, pp. 447-459, 2012.

[12] K. H. Sang, S. J. Park, J. Wu et al., "Temperature variation in steel box girders of cable-stayed bridges during construction," Journal of Constructional Steel Research, vol. 112, pp. 80-92, 2015.

[13] J. Ye, L. Jia, and P. Qian, "Observation andresearch on temperature distribution in concrete boxgirders," Journal of Southeast University (Natural Science Edition), vol. 32, no. 5, pp. 788-793, 2002.

[14] P. Ribeiro and E. Manoach, "The effect of temperature on the large amplitude vibrations of curved beams," Journal of Sound and Vibration, vol. 285, no. 4/5, pp. 1093-1107, 2005.

[15] Z. Pang, X. Xu, S. Xing et al., "Analysis of temperature field and temperature effect of urban concrete curved beam bridge," China Foreign Highway, vol. 36, no. 1, pp. 124-130, 2016.

[16] W. Tan, Z. Zhang, and Z. Ke, "Discussion on temperature deformation and support system arrangement of plane curved beam bridge," Railway Construction, vol. 1, no. 4, pp. 7-9, 2005.

[17] Y. Jiang and Y. Zhang, "Analysis of sunshine temperature effect of curved continuous box girder bridge," Gansu Science and Technology, vol. 30, no. 6, pp. 104-106, 2014.

[18] X. Zhao, X. Xu, X. Li et al., "Study on the influence of temperature change on prestressed concrete curved beam bridge," Highways, vol. 59, no. 11, pp. 42-47, 2014.

[19] M. Mohammadi and J. R. Dryden, "Thermal stress in a nonhomogeneous curved beam," Journal of Thermal Stresses, vol. 31, no. 7, pp. 587-598, 2008.

[20] N. S. Rajabzadeh, S. M. Hozhabrossadati, and M. P. Rezaiee, "Three-dimensional deformations of a curved circular beam subjected to thermo-mechanical loading using green's function method," International Journal of Mechanical Sciences, vol. 142, pp. 163-175, 2018.

[21] Y. . and J. Ye, "Computing method of effect of thermal gradient on concrete curved box-girders," Journal of Southeast
University(Natural Science Edition, vol. 35, no. 6, pp. 924-929, 2005.

[22] C. Fu and L. I. Qiao, "Torsion analysis of horizontal curved box girder under temperature difference," Journal of Southwest Jiaotong University, vol. 44, no. 5, pp. 710-715, 2009.

[23] China Communications Highway Planning and Design Institute, General Code for Design of Highway Bridges and Culverts (JTG D60-2015), People's Communications Press, Beijing, China, 2015.

[24] China Railway Engineering Design Consulting Group Co., Ltd, Code for Design of Concrete Structures of Railway Bridges and Culverts (TB 10092-2017), China Railway Press, Beijing, China, 2017. 